\title{
Towards industrialization of graphene oxide
}

\author{
Yufei Ma ${ }^{1}$, Yaxuan Zheng ${ }^{1}$ and Yanwu $\mathrm{Zhu}^{2^{*}}$
}

\begin{abstract}
Graphene oxide (GO) has demonstrated potential applications in various fields, and attracted intensive attention in industry as well. Numerous companies worldwide have been working on the industrial applications of GO-based materials in, e.g., thermal management, multifunctional composites, anti-corrosion paints, lubricants, energy storage, environment protection and biomedicals. This review presents a short summary on the proceedings of GO towards industrialization, including the large-scale production and some promising applications, by providing views on the processing strategies and challenges specifically for the industrial use of GO. This review would help the scientists in this area to find topics for overcoming challenges together with engineers.
\end{abstract}

Keywords: graphene oxide, mass production, industrial application

\section{INTRODUCTION}

Graphene oxide (GO), a monolayer analog to graphene but with rich oxygen-containing groups on the carbon skeleton [1-3], has witnessed an intensive research boom worldwide in the past decade. GO has been considered as one of the most promising precursors to produce graphene materials at industrial scale and other applications of interest for several reasons. First, single-layered GO platelets can be readily manufactured through the exfoliation of graphite oxide in numerous solvents. This has triggered a huge amount of research on the assembly of such nanoplatelets to macroscopic structures such as fibers, films and aerogels. Secondly, the abundant functional groups on GO allow versatile chemical modifications for a wide range of applications, especially for hybridizing GO. Thirdly, the excellent dispersibility of $\mathrm{GO}$ in water and some organic solvents makes the processing based on the current chemical engineering facility possible, which is critical to constantly lower the cost of GO. In addition, the de-oxidization (typically called reduction) of GO, which is often needed to partially recover the conjugate structure and some properties (e.g., electrical or thermal conductivity), can be carried out simultaneously with the processing mentioned above under many circumstances, increasing the flexibility of GO used in final products. Therefore, numerous companies are encouraged to enter GO-related markets to commercialize the preparation and some applications proposed by scientists.

Understanding the structure of GO is essential for the appropriate utilization of GO. Generally, GO is composed of carbon, oxygen and hydrogen atoms, among which the oxygen atom is mainly in the form of carboxyl, hydroxyl, ketone and epoxy groups. With magic angle spinning nuclear magnetic resonance (MAS NMR), Lerf and Klinowski (LK) model [4] demonstrated that the epoxy and hydroxyl groups were on the GO plane, while carboxyl and hydroxyl groups were at the edges. In LK model, two kinds of carbon domains are present in GO: $\mathrm{sp}^{2}$ carbon domain which remains intact as in graphene and $\mathrm{sp}^{3}$ carbon domain consisting of oxygen groups, which are verified by high resolution transmission electron microscopy (HRTEM) [5,6]. Tour et al. [7] proposed a dynamic model, in which water constantly reacted with $\mathrm{C}=\mathrm{C}$ bonds adjacent to hydroxyl groups to produce acidic groups, explaining why the acidity of a typical GO suspension increased in the second titration. Rourke et al. [8] suggested another "two-component structural model", in which one part of the graphitic structure was slightly oxidized, with the other small polycyclic molecules bearing most of the oxygen functional groups. Although several models are proposed, there is still lack of a clear understanding of the precise structure of $\mathrm{GO}$, as the

\footnotetext{
${ }^{1}$ The Sixth Element (Changzhou) Materials Technology Co., Ltd., Changzhou 213100, China

${ }^{2}$ Hefei National Research Center for Physical Sciences at the Microscale, Department of Materials Science and Engineering, CAS Key Laboratory of Materials for Energy Conversion, iChEM, University of Science and Technology of China, Hefei 230026, China

* Corresponding author (email: zhuyanwu@ustc.edu.cn)
} 
structure of GO is sensitive to the graphite source, preparing technique, processing, preserving conditions and so on. It is a double-edged sword for GO industry: the facile stoichiometry may cause big challenges in largescale preparation and applications of GO, while the specific features of certain GO products may help the manufacturers to set up barriers for potential competitions which is good for marketing specialization.

\section{LARGE SCALE PREPARATION}

The preparation of GO can be tracked to as early as 1859 , when Brodie [9] reported the oxidation of graphite with potassium chlorate $\left(\mathrm{KClO}_{3}\right)$ in fuming nitric acid $\left(\mathrm{HNO}_{3}\right)$. The product was named as "graphitic acid", because it aggregated at acidic $\mathrm{pH}$ but could be dispersed in neutral or basic solution, but he was unaware that this treatment could lead to single-atom layers. With modern analytical techniques, currently $\sim 40 \%$ of graphitic structure with more than $70 \%$ of carbons maintained can be realized when graphite is treated with Brodie's method, and the product mainly contains hydroxyl and epoxy groups [10]. In order to reduce the risk of explosion, Staudenmaier [11] processed graphite by slowly adding $\mathrm{KClO}_{3}$ in portions, into a mixture of fuming $\mathrm{HNO}_{3}$ and sulfuric acid $\left(\mathrm{H}_{2} \mathrm{SO}_{4}\right)$. These two methods, however, are barely used because $\mathrm{KClO}_{3}$ is highly explosive with toxic chlorine dioxide $\left(\mathrm{ClO}_{2}\right)$ formed in the reaction. Hummers and Offeman [12] developed a relatively safer method which has been widely used nowadays. Potassium permanganate $\left(\mathrm{KMnO}_{4}\right)$ is slowly added into a suspension of graphite and sodium nitrate $\left(\mathrm{NaNO}_{3}\right)$ in concentrated $\mathrm{H}_{2} \mathrm{SO}_{4}$ at a temperature lower than $20^{\circ} \mathrm{C}$; then the suspension is kept at $35^{\circ} \mathrm{C}$ for the oxidation, and finally diluted with water at $98^{\circ} \mathrm{C}$ to terminate the oxidation. As shown in Fig. 1, during the reaction, $\mathrm{H}_{2} \mathrm{SO}_{4}$ molecules diffuse into graphite in several minutes and form a graphite intercalated compound (GIC), before the active oxidants $\left(\mathrm{Mn}_{2} \mathrm{O}_{7}\right.$ or $\left.\mathrm{MnO}_{3}^{+}\right)$react with graphitic layers [13]. The control step of the reaction is the diffusion of oxidants through the layers, therefore the efficiency of oxidation is determined by the reaction time [14] and the characteristics of graphite, such as crystallization [15], thickness and particle size [16]. The diffusion-controlled oxidation also makes it challenging to obtain large GO flakes [17], because the exfoliated layers may be further oxidized and cut into smaller pieces [18]. Tour et al. [19] demonstrated an improved Hummers' method for an increased number of isolated aromatic rings in $\mathrm{GO}$ by using the mixture of phosphoric acid $\left(\mathrm{H}_{3} \mathrm{PO}_{4}\right)$ and $\mathrm{H}_{2} \mathrm{SO}_{4}$ $\left(v_{\mathrm{H}_{3} \mathrm{PO}_{4}}: v_{\mathrm{H}_{2} \mathrm{SO}_{4}}=1: 9\right)$, in which the formation of five-membered cyclic phosphate groups was proposed to prevent further oxidation. But the use of $\mathrm{H}_{3} \mathrm{PO}_{4}$ hampers its potential application in industry as the phosphorus pollution in water may cause dense plant growth and severe eutrophication. Another strategy of reducing the oxidative cutting is to apply a pre-oxidation followed by a mild oxidation. For example, Lu et al. [20] utilized $\mathrm{CrO}_{3}$ and hydrochloric acid $(\mathrm{HCl})$ to produce chemically expanded graphite (CEG), which was highly accessible (surface area $>800 \mathrm{~m}^{2} \mathrm{~g}^{-1}$ ) to the sequential oxidants and thus effectively boosted the mass diffusion. With low oxidant usage (2 times of graphite mass), GO platelets with an average size of $128 \mu \mathrm{m}$ and a yield of $\sim 100 \%$ were obtained.

In addition to optimizing conventional methods, researchers have also developed other strategies for more efficient oxidization of graphite. Gao et al. [21] reported an iron-based method to prepare $\mathrm{GO}$ within $1 \mathrm{~h}$, in which the use of $\mathrm{K}_{2} \mathrm{FeO}_{4}$ oxidant avoided the pollution of heavy metals (e.g., $\mathrm{Mn}$ ) and enabled the recycling of $\mathrm{H}_{2} \mathrm{SO}_{4}$. Yu et al. [22] further combined $\mathrm{K}_{2} \mathrm{FeO}_{4}$ with $\mathrm{KMnO}_{4}$ for an improved intercalation and pre-oxidation of graphite, and then fed another portion of $\mathrm{KMnO}_{4}$ for the deeper oxidation. The reliability of $\mathrm{K}_{2} \mathrm{FeO}_{4}$, however, has been argued by Sofer et al. [23] after they replicated and evaluated the oxidation with $\mathrm{K}_{2} \mathrm{FeO}_{4}$. Other methods, e.g., electrochemical oxidation, are also considered as alternatives for the production of GO. Pumera et al. [24] applied a voltage of $10 \mathrm{~V}$ to a graphite foil in the mixture
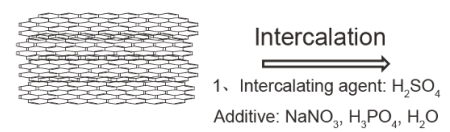

Graphite Additive: $\mathrm{NaNO}_{3}, \mathrm{H}_{3} \mathrm{PO}_{4}, \mathrm{H}_{2} \mathrm{O}$

- Intercalating agent

- : Oxidizing agent

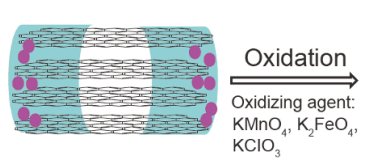

GIC

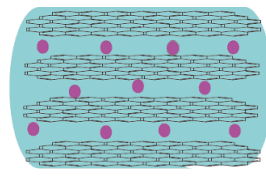

Graphite oxide

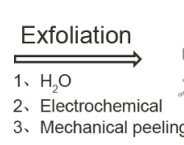

3. Mechanical peeling

GO sheets

Figure 1 Strategies for GO preparation. In a typical process, graphite is firstly intercalated with $\mathrm{H}_{2} \mathrm{SO}_{4}$ and/or other chemicals to form GIC. Then GIC is oxidized to graphite oxide with chemical reagents such as $\mathrm{KMnO}_{4}$ or through electrochemical oxidation. Graphite oxide is then exfoliated to GO platelets with the assistance of sonication or strong shearing forces in proper solvents. 
of $\mathrm{H}_{2} \mathrm{SO}_{4}, \mathrm{Na}_{2} \mathrm{SO}_{4}$, and $\mathrm{LiClO}_{4}$, and the resultant product showed a C/O ratio close to 4.0. Cao et al. [25] reported a two-step intercalation and oxidation for the production of GO with an oxygen content of 17.7 wt.\%, by sequentially applying $2.2 \mathrm{~V}$ on a commercial graphite foil in concentrated $\mathrm{H}_{2} \mathrm{SO}_{4}$ (> 95\%) for the formation of GIC and $10 \mathrm{~V}$ for the exfoliation in $0.1 \mathrm{~mol} \mathrm{~L}^{-1}\left(\mathrm{NH}_{4}\right)_{2} \mathrm{SO}_{4}$. Pei et al. [26] employed a direct current (DC) of $1.6 \mathrm{~V}$ for the intercalation of graphite foil in $98 \% \mathrm{H}_{2} \mathrm{SO}_{4}$, and then the GIC was oxidized with a DC of $5 \mathrm{~V}$ in $50 \%$ of $\mathrm{H}_{2} \mathrm{SO}_{4}$, followed by sonication for complete exfoliation. In this way $\mathrm{H}_{2} \mathrm{SO}_{4}$ mainly acts as a control to tune the oxygen evolution reaction (OER) of water for the oxidation of graphitic lattice and can be recycled with no other oxidants involved. To completely avoid the use of $\mathrm{H}_{2} \mathrm{SO}_{4}$, $\mathrm{Lu}$ et al. [27] demonstrated a bio-electrochemical method with the help of bacterial community, from which 1-3 layers of GO with a lateral size of 300-500 nm were obtained at room temperature in about 2 months. The microorganisms were considered to reduce the overpotential of reactions on the electrode in "digesting" graphite.

For an industrial-scale preparation, several issues other than those in labs need to be considered. First, the quality of GO should be constantly guaranteed, which requires a constant quality of all raw materials, a strict control over the whole procedures, reliable assessments for each processing step and the final materials, and even comprehensive plans for the storage and transportation of GO. Secondly, the manufacturing cost of GO should be further reduced to below $100 \mathrm{RMB} \mathrm{kg}^{-1}$, for the wider range of applications. To realize this target, engineers desire the higher oxidative efficiency, the simpler purification technique, and highly efficient processing schemes and related facility. Thirdly, safety and environmental issues are extremely important for the industrial-level production of GO. Toxicity, flammability and explosibility of all materials with daily exposure of workers, resultant chemical pollutants and noise during the production, and recycling of substance and energy should be carefully evaluated prior to any large-scale production plans. Another aspect related to GO preparation is the reduction of $\mathrm{GO}$ for better electrical conductivity. Among various techniques to remove oxygen groups, thermal treatment has been considered as the most accessible approach for reduced GO ( $\mathrm{GGO}$ ), although the release of gas such as $\mathrm{CO}_{2}$ from carboxyl groups may leave defects or holes on rGO [28]. The rapid evaporation of water absorbed on GO may cause wrinkled structure and agglomeration of rGO platelets [29]. Ideally, the synthetic procedure of GO or rGO should be highly tunable to meet the requirements in different applications.

\section{INDUSTRIAL APPLICATOINS}

Encouraged by many fantastic properties of graphene, a wide range of applications related to GO have been explored towards the commercialization. To utilize GO in products, one strategy is to assemble GO platelets into macroscopic products; the other one is to integrate GO with some matrix materials for the improvement of certain functions. Herein, some emerging industrial applications based on GO are briefly reviewed, with each towards potentially a huge market and significant effect on the specific industry.

\section{Thermal management}

As graphene intrinsically owns very high thermal conductivity, naturally films made from GO have been considered as a platform for heat dissipation. As shown in Fig. 2, to achieve a high thermal transfer power, GO is typically stacked into highly-ordered micrometer-thick films which are freestanding and ready for the following processing. Heat treatment at high temperatures is needed to remove oxygen (and some other impurities), and to repair the defects of GO for high thermal conductivity. For example, a treatment at $1000^{\circ} \mathrm{C}$ has increased the thermal conductivity from $\sim 3$ to $\sim 61 \mathrm{~W} \mathrm{~m}^{-1} \mathrm{~K}^{-1}$ for a $40 \mu \mathrm{m}$-thick GO film [30], due to the decrease of defects concentration. It is worth noting that a much higher thermal conductivity of $1043 \mathrm{~W} \mathrm{~m}^{-1} \mathrm{~K}^{-1}$ was reported by increasing the annealing temperature to $1200^{\circ} \mathrm{C}$ [31]. The in-plane thermal conductivity of GO-based films can be further improved by engineering the grain size, film alignment and thickness, and interlayer binding energy in GO stacking [32]. It has been demonstrated that a dense $\left(\sim 2.1 \mathrm{~g} \mathrm{~cm}^{-3}\right)$, thin $(0.8 \mu \mathrm{m})$, large-grain $(13.3 \mu \mathrm{m})$, and partly turbostratic-stacking (37\%) film made from GO could benefit the transfer of both high-frequency diffusive phonons and low-frequency ballistic phonons, leading to a superior thermal conductivity of $3200 \mathrm{~W} \mathrm{~m}^{-1} \mathrm{~K}^{-1}$ [32].

The films made from GO has been applied to heat dissipation for devices. Microscale patterns of GO were fabricated on a sapphire substrate, followed by the reduction and an epitaxial lateral overgrowth of gallium nitride $(\mathrm{GaN})$ via a metal-organic chemical vapor deposition (MOCVD) [33]. The embedded rGO in the GaN light-emitting diode (LED) has alleviated the self-heating issues evidenced by the decreased temperature on the chip surface from 51.4 to $47^{\circ} \mathrm{C}$. When a graphene-based film was bonded to functionalized GO through amino- 


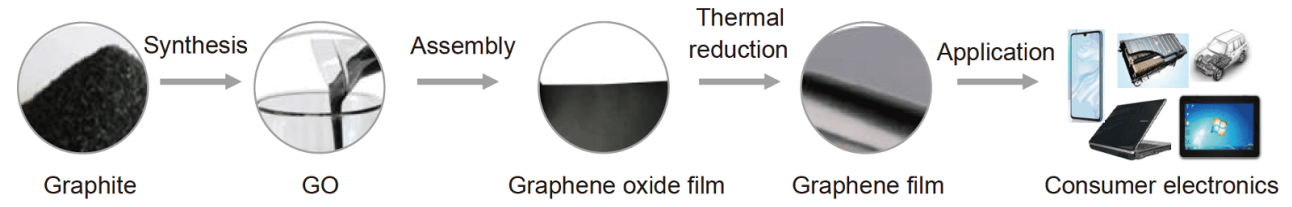

Figure 2 Typical preparation for the production of thermal dissipation films.

silane molecules as alternative heat-escaping channels, the hotspot temperature of a micro heater operating at $1300 \mathrm{~W} \mathrm{~cm}^{-2}$ was lowered by $\sim 28^{\circ} \mathrm{C}$ [34]. Huawei Inc. has launched mobile phones using GO-derived heat dissipating films combined with fluid channels [28].

\section{Multifunctional composites}

Graphene is considered to improve the mechanical, electrical or thermal properties as additives in composites. In many situations, the dispersibility of graphene platelets in the composites is challenging, as the intrinsic graphene itself tends to agglomerate due to the van de Walls interaction between layers. In contrast, GO, with tunable functional groups, is compatible with matrix materials such as polymers. The following several examples are taken to demonstrate the potential of GO for multifunctional composites.

Polyamide 6 (PA6) is an important engineering plastic. It was reported that $\mathrm{GO}$ with small lateral size $(\sim 0.42 \mu \mathrm{m})$ could be well dispersed in PA6 fibers via melting spinning [35], and thus substantially improve the mechanical properties of composite fibers. GO has also been incorporated with silica nanoparticles via Fischer esterification [36], as a co-filler in PA6 composites which demonstrate remarkable improvement in stiffening (up to $180 \%$ ), strengthening (up to $210 \%$ ) and toughening (up to $210 \%$ ) at low contents (no more than $0.5 \mathrm{wt} . \%$ ) of GO/ $\mathrm{SiO}_{2}$ filler. The dispersion of GO or $\mathrm{rGO}$ is a big challenge in the post-processing because of the high viscosity of PA6. A pre-dispersion of GO in solvent was developed before the melt processing, and it was found that the filler dispersion was the most crucial parameter affecting ultimate properties, as the mobility of macromolecules was evidently restricted by the GO network [37]. In contrast, in-situ polymerization with the existence of $\mathrm{GO} / \mathrm{rGO}$ seems more attractive as $\mathrm{GO} / \mathrm{rGO}$ can be dispersed in monomers more uniformly. For example, no re-stacking was observed during the in-situ polymerization of $\varepsilon$-caprolactam and the reduction of GO simultaneously [38]. The presence of GO has promoted the nucleation of PA6 crystallites due to the bonding between polymer chains and GO surface, thus improving the thermal stability of
PA6 [39]. After the thermal reduction during polymerization, an ultralow percolation threshold (0.11 vol.\%) in electrical conductivity of PA6 is achieved [40].

When being incorporated into polymers for coating, $\mathrm{GO} / \mathrm{rGO}$ can improve the anti-corrosion performance. Electrochemical impedance spectroscopy (EIS) analysis has shown that self-aligned $\mathrm{rGO}$ in waterborne polyurethane (PU) coated on an electrogalvanized steel improves the anti-corrosion performance [41]. Polyisocyanate (PI) resin was covalently attached to the surface of GO through the formation of amides and carbamate esters bonds [42], leading to a significant improvement of the coating corrosion protection with only $0.1 \mathrm{wt} . \% \mathrm{GO}$ in the PU. Similarly, with the addition of $0.1 \mathrm{wt} . \%$ amino-functionalized GO, the deterioration of the epoxy resin was decreased because GO might react with hydroxyl ions and prevent the $\mathrm{Cl}$ ions to diffuse into the coating [43]. The idea was realized in industry and applied to a wind mill tower in 2015 [44]. As shown in Fig. 3, The Sixth Element (Changzhou) Materials Technology Co., Ltd. produced a 2-component epoxy primer (25 wt.\% zinc powder and $1 \mathrm{wt} . \%$ rGO) that fulfilled all requirements for highly corrosive environments, and demonstrated that the primer alone could withstand more than $3000 \mathrm{~h}$ in the harsh environment with a dryfilm thickness of $50 \mu \mathrm{m}$ in standard salt-spray testing. Compared to the situation with $70 \mathrm{wt} . \% \mathrm{Zn}$, the presence of rGO has efficiently bridged $\mathrm{Zn}$ particles and prevented the diffusion of corrosive electrolyte to the surface of steel as well.

\section{Energy and environmental applications}

Graphene has attracted intense interest in energy storage and this topic has been reviewed in many articles [45-49]. GO-derived carbons have been used as active materials for storing charges in a double-layer (as in electrochemical double-layer capacitors, EDLCs) [50], as media to host ions (such as $\mathrm{Li}^{+}$or $\mathrm{Na}^{+}$in ion batteries) [51], or as a catalyst in metal-air batteries [52]. In many reports, GO has been assembled into 3D structures to achieve a large surface area or to combine with another active materials and the following reduction makes $\mathrm{GO}$ more 


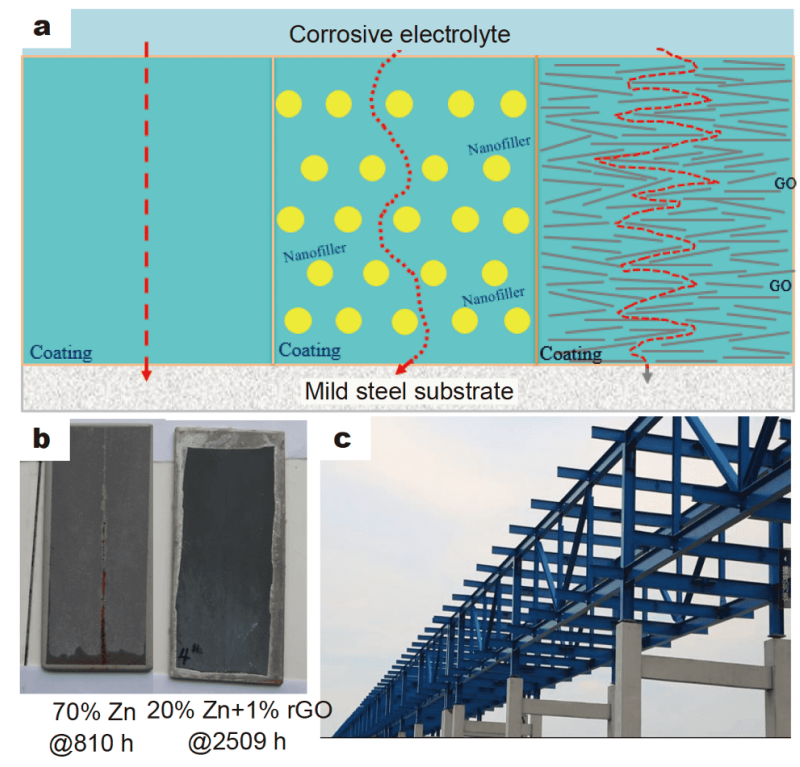

Figure 3 (a) Schematic of GO dispersed in a coating layer, compared with spherical nanofillers or without coating; (b) salt spray testing indicates the better anti-corrosive performance for rGO-containing epoxy primer compared with conventional primer; (c) a rack in Nantong, China, applied with rGO-enhanced anti-corrosive primer.

conducting. In addition, GO or rGO can be used as ink for printing, largely broadening the use of GO-based materials in energy storage [53]. One practical usage of $\mathrm{GO}$ for energy storage is in cathodes of Li-ion batteries, after GO is reduced and dispersed in $\mathrm{N}$-methyl pyrrolidone (NMP), and a combination of rGO with carbon nanotubes or carbon black is often needed.

GO has shown potential in optimizing the performance of fuel cells especially in cathodes and in separation membranes [54]. Sulfonated GO has been added into polybenzimidazole (PBI) for high-temperature polymer electrolyte membrane fuel cells with $\mathrm{H}_{3} \mathrm{PO}_{4}$ to enhance the proton conductivity, and a power density of $600 \mathrm{~mW} \mathrm{~cm}^{-2}$ at $175^{\circ} \mathrm{C}$ was achieved [55]. The sulfonic group has been considered as a permeation channel for protons and GO platelets blocks water and methanol molecules, as illustrated in sulfonated $\mathrm{GO} /$ sulfonated polyether ether ketone (PEEK) composite membrane [56]. A graphene-modified polyvinyl alcohol silicotungstic acid membrane was used for methanol fuel cells, in which the power density was improved from 0.88 to $1.19 \mathrm{~W} \mathrm{~m}^{-3}$ for single-cell with Nafion 117 [57]. The connected and continuous ionic channels of GO have brought enhanced ionic conductivity by $\sim 126 \%$ and increased power density by $\sim 148 \%$, in a poly(vinyl alcohol)/ GO membrane with 0.7 wt.\% GO loading [58].
The GO film has shown potential to directly generate power which is sensitive to moisture variation, due to the gradient process of oxygen-containing groups [59]. The device was able to provide a moisture-enabled voltage output of $\sim 35 \mathrm{mV}$ and a power of $\sim 4.2 \mathrm{~mW} \mathrm{~m}^{-2}$ with an energy conversion efficiency of up to $\sim 62 \%$, by harvesting energy from moisture diffusion. The energy comes from the self-ionization of functional groups in response to moisture and thus an ion concentration gradient [60], which can be eliminated by recombination of anions and cations. This phenomenon has been used to fabricate a stacking device consisting of 60 units, which could produce an electric voltage of $11.2 \mathrm{~V}$ in ambient air [61].

Graphene-based materials have also emerged as potential candidates for desalination. The space between GO layers may act as the interconnected nanochannels for water permeation, which can be tuned through the creation of stabilizing force between the layers [62,63]. A high water flux that is theoretically $2-3$ orders of magnitude higher than the current commercial reverse osmosis (RO) membrane [64] is obtained and a controllable distribution of the hydrophobic region in GO facilitates the fast water permeation. Nair et al. [65] demonstrated that the GO film allowed a unique water permeation pathway but selectively hindered the motion of gases and non-aqueous solutions. A classical molecular dynamics shows that the desalination ability of GO membranes is sensitive to the pore diameter and hydroxyl groups in the membrane, as the hydrophilic feature would improve the accessibility to water [64]. With the help of potassium ions, Chen et al. [63] demonstrated that the interlayer spacing of GO film could be precisely controlled with a resolution down to $1 \AA$ A enabling an efficient ion rejection. The light absorption ability of GO/rGO has been utilized with the confined water path in a solar desalination device, based on which the heat generated from solar energy could evaporate the water passing the layers while the salt being separated, enabling an efficient $(80 \%$ under onesun illumination) and effective (four orders salinity decrement) solar desalination [66].

With large surface areas and tunable structure/functional groups, GO-based materials have been used for water treatment, through filtering, adsorption, and/or photodegradation [67]. A removal rate of over $95 \%$ for organic molecules such as safranin dye [68], methylene blue [69], ibuprofen drug [70], as well as an adsorption capacity of $10-400 \mathrm{mg} \mathrm{g}^{-1}$ for heavy metal ions has been achieved [71]. Furthermore, GO has been combined with variety of metal oxides including $\mathrm{TiO}_{2}, \mathrm{ZnO}, \mathrm{Cu}_{2} \mathrm{O}$, $\mathrm{ZnFe}_{2} \mathrm{O}_{4}, \mathrm{CuFe}_{2} \mathrm{O}_{4}$ and $\mathrm{Bi}_{2} \mathrm{WO}_{6}$ for the degradation of 
synthetic dyes as excellent photocatalysts [71]. In such composites, the dispersibility of GO plays important role for coupling the catalysts and transferring electrons.

\section{Other potential applications}

Other than those above-mentioned applications, GO has also been extensively studied for various potential biomedical applications, such as drug delivery, biosensing and monitoring, cancer treatment via stimuli-responsive heating, tissue engineering and antimicrobial activities. The safety issues, however, are still controversial, despite most studies have shown no noticeable impact of GO on the mice and rats, either in long-term or on acute toxicities. The antimicrobial performance of GO may be the most accessible application in a short term, as the antimicrobial effect of graphene materials has been clarified [72]. GO-based bandage [73] and dental care [74] have been used to demonstrate the effect of GO coating [75].

Graphene can be used as a self-lubricating solid or an additive for lubricating oils [76]. GO films with tunable thickness were coated onto a silicon wafer via electrophoretic deposition (EPD), and the friction coefficient of the silicon wafer and the wear volume were reduced to $1 / 6$ and $1 / 24$ of the original value, respectively [77]. The rGO balls, made by spray-drying $\mathrm{GO}$ at $400^{\circ} \mathrm{C}$ and subsequent thermal reduction at $700^{\circ} \mathrm{C}$ [29], could be dispersed in polyalphaolefin based oil. The oil with a $\mathrm{rGO}$ concentration of $0.01-0.1 \mathrm{wt} . \%$ has outperformed a fully formulated commercial 5W30 lubricant in terms of friction and wear reduction. When rGO was chemically linked with polyethylene glycol (PEG) molecules through hydrogen bonding, $0.2 \mathrm{mg} \mathrm{mL}^{-1}$ of $\mathrm{rGO}$ in the lube medium showed a friction coefficient of 0.06 and reduced wear of up to $50 \%$ compared with bare PEG lubricated steel-steel contact [78]. In another report, two different rGO samples terminated by hydroxyl or epoxy-hydroxyl groups were compared and a significantly lower friction coefficient was observed with the epoxy-hydroxyl-terminated rGO [79]. The results are ascribed to the intercalation of PEG molecules between the basal planes of the graphene through epoxy-hydroxyl interactions between rGO and PEG.

\section{PERSPECTIVE AND OUTLOOK}

Based on extensive studies on GO and its applications, the mass production of GO has been realized by several companies $[28,80]$, and some GO-based products also emerged on the market. The GO-related industry, however, is still exploring and enlarging its market while optimizing GO-derived products according to specific requirements is desired. Herein we summarize some challenges in GO commercialization.

(i) People have been struggling with the uncontrolled and costly preparation of GO since its emergence, which is the key for large-scale production and industrial use. Specifically for Hummers' method that dominates the synthetic strategies in either academia or industry, the oxidation process remains a black box although several mechanisms were proposed. As a result, fine control over the functional groups and size of GO is challenging, and thus the classification of GO is difficult for further identification of the key characteristics in applications. In addition, much effort is needed for the efficient use of oxidative agents, dispersant medium, purifying solvents, other chemical resources and energy. The production cost of GO remains high compared with many fundamental raw materials and hinders its further applications in many areas.

(ii) There is lack of standardization for graphene materials including GO in the market, which is closely related to the issues in characterizations. GO or rGO could be tested via electron microscopy, atomic force microscopy, Raman spectroscopy, elemental analysis, X-ray photoelectron spectrometry, and scanning and transmission electron microscopy, but the most evaluations are time- or cost-consuming for industrial users. Seeking for simple, fast, reliable methods on accessible equipment is especially important for small or start-up companies. In particular, the identification of $\mathrm{GO} / \mathrm{rGO}$ in matrix is challenging because of the typically low dosage (e.g., 0.01-1 wt.\%), and undistinguished interface between GO and polymer matrix in many situations.

(iii) Controlled reduction and modification of GO in large scale are still far from rational. Especially when GO/ rGO serves as an additive, the performance of the composites greatly relies on the dispersibility of $\mathrm{GO} / \mathrm{rGO}$. Despite of numerous reports about the functionalization of GO via wet chemistry, the concentration of GO for the reaction is typically low to prevent crosslinking and/or reagglomeration, and in-situ reduction and modification in the matrices are seldom developed, which affects the original process such as polymerizations. Developing high concentration GO-based products with tunable geometrical and chemical features is desired for competing with other widely used additives in the market.

(iv) Safety issues of GO-related materials, especially chronic toxicity and genotoxicity, require further investigation. As existing toxicity studies indicate, the in vitro and in vivo toxicity of GO are dependent on its purity, size, oxygen-containing groups, surface chemistry, 
and evaluation models. These studies imply an uncertainty of safety issue related to GO when a GO sample is not fully recognized in its chemical structure and morphology. Furthermore, few studies addressed the genotoxicity of GO materials, and very limited for chronic toxicity. This situation is disadvantageous for safety registration prior to marketing GO-based products, especially when GO is directly exposed in vivo, such as drug delivery and tissue engineering.

\section{Received 1 June 2019; accepted 19 June 2019;} published online 9 July 2019

1 Dreyer DR, Park S, Bielawski CW, et al. The chemistry of graphene oxide. Chem Soc Rev, 2010, 39: 228-240

2 Chen D, Feng H, Li J. Graphene oxide: Preparation, functionalization, and electrochemical applications. Chem Rev, 2012, 112: 6027-6053

3 Bianco A, Cheng HM, Enoki T, et al. All in the graphene family-A recommended nomenclature for two-dimensional carbon materials. Carbon, 2013, 65: 1-6

4 He H, Klinowski J, Forster M, et al. A new structural model for graphite oxide. Chem Phys Lett, 1998, 287: 53-56

5 Gomez-Navarro C, Meyer JC, Sundaram RS, et al. Atomic structure of reduced graphene oxide. Nano Lett, 2010, 10: 1144-1148

6 Erickson K, Erni R, Lee Z, et al. Determination of the local chemical structure of graphene oxide and reduced graphene oxide. Adv Mater, 2010, 22: 4467-4472

7 Dimiev AM, Alemany LB, Tour JM. Graphene oxide. Origin of acidity, its instability in water, and a new dynamic structural model. ACS Nano, 2013, 7: 576-588

8 Rourke JP, Pandey PA, Moore JJ, et al. The real graphene oxide revealed: Stripping the oxidative debris from the graphene-like sheets. Angew Chem, 2011, 123: 3231-3235

9 Brodie BC. XIII. On the atomic weight of graphite. Philosophical Transactions of the Royal Society of London, 1859, 149: 249-259

10 Botas C, Álvarez P, Blanco P, et al. Graphene materials with different structures prepared from the same graphite by the Hummers and Brodie methods. Carbon, 2013, 65: 156-164

11 Staudenmaier L. Verfahren zur Darstellung der Graphitsäure. Ber Dtsch Chem Ges, 1898, 31: 1481-1487

12 Hummers Jr. WS, Offeman RE. Preparation of graphitic oxide. J Am Chem Soc, 1958, 80: 1339

13 Dimiev AM, Tour JM. Mechanism of graphene oxide formation. ACS Nano, 2014, 8: 3060-3068

14 Jeong HK, Jin MH, So KP, et al. Tailoring the characteristics of graphite oxides by different oxidation times. J Phys D-Appl Phys, 2009, 42: 065418

15 Botas C, Álvarez P, Blanco C, et al. The effect of the parent graphite on the structure of graphene oxide. Carbon, 2012, 50: 275282

16 Chen J, Li Y, Huang L, et al. High-yield preparation of graphene oxide from small graphite flakes via an improved Hummers method with a simple purification process. Carbon, 2015, 81: 826834

17 Dong L, Yang J, Chhowalla M, et al. Synthesis and reduction of large sized graphene oxide sheets. Chem Soc Rev, 2017, 46: 73067316
18 Pan S, Aksay IA. Factors controlling the size of graphene oxide sheets produced via the graphite oxide route. ACS Nano, 2011, 5: 4073-4083

19 Marcano DC, Kosynkin DV, Berlin JM, et al. Improved synthesis of graphene oxide. ACS Nano, 2010, 4: 4806-4814

20 Dong L, Chen Z, Lin S, et al. Reactivity-controlled preparation of ultralarge graphene oxide by chemical expansion of graphite. Chem Mater, 2017, 29: 564-572

21 Peng L, Xu Z, Liu Z, et al. An iron-based green approach to 1-h production of single-layer graphene oxide. Nat Commun, 2015, 6: 5716

$22 \mathrm{Yu} \mathrm{H}$, Zhang B, Bulin C, et al. High-efficient synthesis of graphene oxide based on improved Hummers method. Sci Rep, 2016, 6: 36143

23 Sofer Z, Luxa J, Jankovský O, et al. Synthesis of graphene oxide by oxidation of graphite with ferrate(VI) compounds: Myth or reality? Angew Chem Int Ed, 2016, 55: 11965-11969

24 Ambrosi A, Pumera M. Electrochemically exfoliated graphene and graphene oxide for energy storage and electrochemistry applications. Chem Eur J, 2016, 22: 435

25 Cao J, He P, Mohammed MA, et al. Two-step electrochemical intercalation and oxidation of graphite for the mass production of graphene oxide. J Am Chem Soc, 2017, 139: 17446-17456

26 Pei S, Wei Q, Huang K, et al. Green synthesis of graphene oxide by seconds timescale water electrolytic oxidation. Nat Commun, 2018, 9: 145

27 Lu L, Zeng C, Wang L, et al. Graphene oxide and $\mathrm{H}_{2}$ production from bioelectrochemical graphite oxidation. Sci Rep, 2015, 5: 16242

28 Zhu Y, Ji H, Cheng HM, et al. Mass production and industrial applications of graphene materials. Natl Sci Rev, 2018, 5: 90-101

29 Dou X, Koltonow AR, He X, et al. Self-dispersed crumpled graphene balls in oil for friction and wear reduction. Proc Natl Acad Sci USA, 2016, 113: 1528-1533

30 Renteria JD, Ramirez S, Malekpour H, et al. Strongly anisotropic thermal conductivity of free-standing reduced graphene oxide films annealed at high temperature. Adv Funct Mater, 2015, 25: 4664-4672

31 Song NJ, Chen CM, Lu C, et al. Thermally reduced graphene oxide films as flexible lateral heat spreaders. J Mater Chem A, 2014, 2 : 16563-16568

32 Wang N, Samani MK, Li H, et al. Tailoring the thermal and mechanical properties of graphene film by structural engineering. Small, 2018, 14: 1801346

33 Han N, Viet Cuong T, Han M, et al. Improved heat dissipation in gallium nitride light-emitting diodes with embedded graphene oxide pattern. Nat Commun, 2013, 4: 1452

34 Han $\mathrm{H}$, Zhang Y, Wang N, et al. Functionalization mediates heat transport in graphene nanoflakes. Nat Commun, 2016, 7: 11281

35 Zhang S, Cheng Y, Xu W, et al. Dispersibility of different sized graphene oxide sheets and their reinforcement on polyamide 6 fibers. RSC Adv, 2017, 7: 56682-56690

36 Scaffaro R, Maio A. A green method to prepare nanosilica modified graphene oxide to inhibit nanoparticles re-aggregation during melt processing. Chem Eng J, 2017, 308: 1034-1047

37 Scaffaro R, Maio A. Optimization of two-step techniques engineered for the preparation of polyamide 6 graphene oxide nanocomposites. Compos Part B-Eng, 2019, 165: 55-64

38 O'Neill A, Bakirtzis D, Dixon D. Polyamide 6/graphene composites: The effect of in situ polymerisation on the structure and 
properties of graphene oxide and reduced graphene oxide. Eur Polymer J, 2014, 59: 353-362

39 Dixon D, Lemonine P, Hamilton J, et al. Graphene oxide-polyamide 6 nanocomposites produced via in situ polymerization. J Thermoplastic Composite Mater, 2015, 28: 372-389

$40 \mathrm{Ma} \mathrm{M}$, Zhu Z, Wu B, et al. Preparation of highly conductive composites with segregated structure based on polyamide- 6 and reduced graphene oxide. Mater Lett, 2017, 190: 71-74

41 Li Y, Yang Z, Qiu H, et al. Self-aligned graphene as anticorrosive barrier in waterborne polyurethane composite coatings. J Mater Chem A, 2014, 2: 14139-14145

42 Ramezanzadeh B, Ghasemi E, Mahdavian M, et al. Covalentlygrafted graphene oxide nanosheets to improve barrier and corrosion protection properties of polyurethane coatings. Carbon, 2015, 93: $555-573$

43 Ramezanzadeh B, Niroumandrad S, Ahmadi A, et al. Enhancement of barrier and corrosion protection performance of an epoxy coating through wet transfer of amino functionalized graphene oxide. Corrosion Sci, 2016, 103: 283-304

44 Münzing B. Greener corrosion protection. Physics World, 2019, 32: $23-25$

45 Wu ZS, Zhou G, Yin LC, et al. Graphene/metal oxide composite electrode materials for energy storage. Nano Energy, 2012, 1: 107131

46 Yang Z, Tian J, Yin Z, et al. Carbon nanotube- and graphene-based nanomaterials and applications in high-voltage supercapacitor: A review. Carbon, 2019, 141: 467-480

47 Li F, Jiang X, Zhao J, et al. Graphene oxide: A promising nanomaterial for energy and environmental applications. Nano Energy, 2015, 16: 488-515

48 Raccichini R, Varzi A, Passerini S, et al. The role of graphene for electrochemical energy storage. Nat Mater, 2014, 14: 271-279

49 Georgakilas V, Tiwari JN, Kemp KC, et al. Noncovalent functionalization of graphene and graphene oxide for energy materials, biosensing, catalytic, and biomedical applications. Chem Rev, 2016, 116: 5464-5519

50 Zhu Y, Murali S, Stoller MD, et al. Carbon-based supercapacitors produced by activation of graphene. Science, 2011, 332: 1537-1541

51 Lin D, Liu Y, Liang Z, et al. Layered reduced graphene oxide with nanoscale interlayer gaps as a stable host for lithium metal anodes. Nat Nanotech, 2016, 11: 626-632

52 Jung HG, Jeong YS, Park JB, et al. Ruthenium-based electrocatalysts supported on reduced graphene oxide for lithium-air batteries. ACS Nano, 2013, 7: 3532-3539

53 Down MP, Rowley-Neale SJ, Smith GC, et al. Fabrication of graphene oxide supercapacitor devices. ACS Appl Energy Mater, 2018, 1: $707-714$

54 Farooqui UR, Ahmad AL, Hamid NA. Graphene oxide: A promising membrane material for fuel cells. Renew Sustain Energy Rev, 2018, 82: 714-733

$55 \mathrm{Xu} \mathrm{C}$, Cao Y, Kumar R, et al. A polybenzimidazole/sulfonated graphite oxide composite membrane for high temperature polymer electrolyte membrane fuel cells. J Mater Chem, 2011, 21: 1135911364

56 Heo Y, Im H, Kim J. The effect of sulfonated graphene oxide on sulfonated poly (ether ether ketone) membrane for direct methanol fuel cells. J Membrane Sci, 2013, 425-426: 11-22

57 Khilari S, Pandit S, Ghangrekar MM, et al. Graphene oxide-impregnated PVA-STA composite polymer electrolyte membrane separator for power generation in a single-chambered microbial fuel cell. Ind Eng Chem Res, 2013, 52: 11597-11606

58 Ye YS, Cheng MY, Xie XL, et al. Alkali doped polyvinyl alcohol/ graphene electrolyte for direct methanol alkaline fuel cells. J Power Sources, 2013, 239: 424-432

59 Zhao F, Cheng $\mathrm{H}$, Zhang Z, et al. Direct power generation from a graphene oxide film under moisture. Adv Mater, 2015, 27: 43514357

60 Zhao F, Wang L, Zhao Y, et al. Graphene oxide nanoribbon assembly toward moisture-powered information storage. Adv Mater, 2017, 29: 1604972

61 Cheng H, Huang Y, Zhao F, et al. Spontaneous power source in ambient air of a well-directionally reduced graphene oxide bulk. Energy Environ Sci, 2018, 11: 2839-2845

62 Joshi RK, Carbone P, Wang FC, et al. Precise and ultrafast molecular sieving through graphene oxide membranes. Science, 2014, 343: $752-754$

63 Chen L, Shi G, Shen J, et al. Ion sieving in graphene oxide membranes via cationic control of interlayer spacing. Nature, 2017, 550: $380-383$

64 Cohen-Tanugi D, Grossman JC. Water desalination across nanoporous graphene. Nano Lett, 2012, 12: 3602-3608

65 Nair RR, Wu HA, Jayaram PN, et al. Unimpeded permeation of water through helium-leak-tight graphene-based membranes. Science, 2012, 335: 442-444

66 Li X, Xu W, Tang M, et al. Graphene oxide-based efficient and scalable solar desalination under one sun with a confined $2 \mathrm{D}$ water path. Proc Natl Acad Sci USA, 2016, 113: 13953-13958

$67 \mathrm{Pu}$ S, Xue S, Yang Z, et al. In situ co-precipitation preparation of a superparamagnetic graphene oxide $/ \mathrm{Fe}_{3} \mathrm{O}_{4}$ nanocomposite as an adsorbent for wastewater purification: Synthesis, characterization, kinetics, and isotherm studies. Environ Sci Pollut Res, 2018, 25: 17310-17320

68 Banerjee P, Sau S, Das P, et al. Optimization and modelling of synthetic azo dye wastewater treatment using graphene oxide nanoplatelets: Characterization toxicity evaluation and optimization using Artificial Neural Network. Ecotoxicol Environ Saf, 2015, 119: $47-57$

69 Song S, Ma Y, Shen H, et al. Removal and recycling of ppm levels of methylene blue from an aqueous solution with graphene oxide. RSC Adv, 2015, 5: 27922-27932

70 Banerjee P, Das P, Zaman A, et al. Application of graphene oxide nanoplatelets for adsorption of ibuprofen from aqueous solutions: Evaluation of process kinetics and thermodynamics. Process Saf Environ Protection, 2016, 101: 45-53

71 Upadhyay RK, Soin N, Roy SS. Role of graphene/metal oxide composites as photocatalysts, adsorbents and disinfectants in water treatment: A review. RSC Adv, 2014, 4: 3823-3851

72 Zou X, Zhang L, Wang Z, et al. Mechanisms of the antimicrobial activities of graphene materials. J Am Chem Soc, 2016, 138: 2064 2077

73 Konwar A, Kandimalla R, Kalita S, et al. Approach to fabricate a compact cotton patch without weaving: A smart bandage material. ACS Sustain Chem Eng, 2018, 6: 5806-5817

74 He J, Zhu X, Qi Z, et al. Killing dental pathogens using antibacterial graphene oxide. ACS Appl Mater Interfaces, 2015, 7: $5605-5611$

75 Li S, Lee JK, Zhou S, et al. Synthesis of surface grown Pt nanoparticles on edge-enriched $\mathrm{MoS}_{2}$ porous thin films for enhancing electrochemical performance. Chem Mater, 2019, 31: 387-397

76 Berman D, Erdemir A, Sumant AV. Graphene: A new emerging 
lubricant. Mater Today, 2014, 17: 31-42

77 Liang $\mathrm{H}, \mathrm{Bu} \mathrm{Y}$, Zhang J, et al. Graphene oxide film as solid lubricant. ACS Appl Mater Interfaces, 2013, 5: 6369-6375

78 Gupta B, Kumar N, Panda K, et al. Energy efficient reduced graphene oxide additives: Mechanism of effective lubrication and antiwear properties. Sci Rep, 2016, 6: 18372

79 Gupta B, Kumar N, Panda K, et al. Role of oxygen functional groups in reduced graphene oxide for lubrication. Sci Rep, 2017, 7: 45030

80 Kauling AP, Seefeldt AT, Pisoni DP, et al. The worldwide graphene flake production. Adv Mater, 2018, 30: 1803784

Acknowledgements The authors acknowledge Yanlin Zhang for her assistance. This work was supported by the National Natural Science Foundation of China (51772282).

Author contributions $\mathrm{Ma} \mathrm{Y}$ and Zhu Y wrote the manuscript; Zheng $\mathrm{Y}$ drew the figures. All authors contributed to the general discussion.

Conflict of interest The authors declare no conflict of interest.

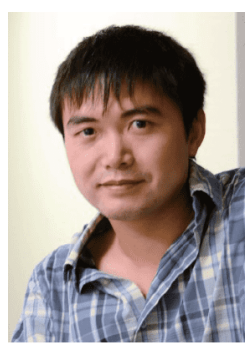

Yufei Ma received his $\mathrm{BSc}$ and $\mathrm{PhD}$ degrees at Lanzhou University in 2006 and 2012, respectively, and he was a visiting graduate student at the University of California, Berkeley during 2010-2012. He worked as a postdoctoral researcher at Suzhou Institute of NanoTech and NanoBionics, Chinese Academy of Sciences, and then joined The Sixth Element (Changzhou) Materials Technology Co., Ltd. in 2015. His research focuses on the industrial production of graphene (oxide) materials and their related applications.

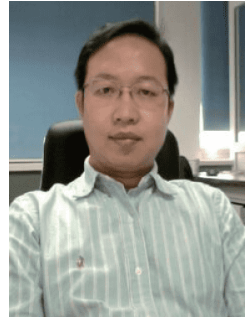

Yanwu Zhu is currently a professor at the Department of Materials Science and Engineering, University of Science and Technology of China. $\mathrm{He}$ received his MSc (2003) from Peking University and $\mathrm{PhD}$ (2007) from the National University of Singapore (NUS), both in physics. He was a postdoctoral researcher at the NUS and the University of Texas at Austin. His research focuses on the preparation, characterization and property research of graphene and other novel carbon materials.

\section{氧化石墨烯的工业化进展}

马宇飞 ${ }^{1}$, 郑雅轩 ${ }^{1}$, 朱彦武 $^{2^{*}}$

摘要 相关研究表明氧化石墨烯(GO)在众多领域皆具有应用潜力, 因此GO越来越受工业界的关注. 目前, 全球已涌现了许多公司, 致 力于实现GO的工业级应用, 如热管理、多功能聚合物复合材料、 防腐、润滑剂、能源、环境和生物医学等. 本文主要介绍了当前 $\mathrm{GO}$ 工业化的发展情况, 包括 GO 的大规模生产以及具有市场经济 价值的应用, 并讨论了 GO在工业化过程中的挑战和处理策略, 旨 在为本领域的科学家提供研究素材, 以期在工业界实现GO的规模 化应用. 